\title{
On the driving force of cation exchange in clays : Insights from combined microcalorimetry experiments and molecular simulation
}

\author{
Benjamin Rotenberg ${ }^{1,2, *}$, Jean-Pierre $\mathrm{Morel}^{3}$, \\ Virginie Marry ${ }^{1,2}$, Pierre Turq ${ }^{1,2}$ and Nicole Morel-Desrosiers ${ }^{3}$ \\ * e-mail: benjamin.rotenberg@upmc.fr$$
\text { tel:+33 (0)1 } 44 \quad 273191
$$$$
\text { fax:+33 (0)1442732 } 28
$$ \\ ${ }^{1}$ CNRS, Laboratoire PECSA, UMR 7195, \\ 4 pl. Jussieu, Paris F-75005, France \\ 2 UPMC-Paris6, Laboratoire PECSA, UMR 7195, \\ 4 pl. Jussieu, Paris F-75005, France and \\ 3 Microorganismes: Génome et Environnement, \\ UMR CNRS 6023, Université Blaise Pascal (Clermont-Ferrand II), \\ 24 avenue des Landais, 63177 Aubière Cedex, France
}




\begin{abstract}
We study the origin of the ionic exchange enthalpy in montmorillonite clays using microcalorimetry measurements and molecular simulation. We first determine the standard reaction enthalpy for well-defined interlayer water contents. We then show by a detailed analysis based on thermodynamic cycles that replacing $\mathrm{Na}^{+}$ions by $\mathrm{Cs}^{+}$in the interlayer of montmorillonite clays is an endothermic process, and that the overall exchange is exothermic only because it is dominated by the exothermic replacement of $\mathrm{Cs}^{+}$by $\mathrm{Na}^{+}$in the aqueous phase. This conclusion from ionic exchange enthalpies supports the one of a recent study of the ionic exchange free energy by Teppen and Miller [Soil. Sci. Soc. Am. J., 70, 31 (2006)] and contradicts long-held views on the role of ion-clay interactions in determining the ionic exchange thermodynamics. This calls for a paradigm shift for the origin of this exchange: The driving force is the "hydrophobicity" of $\mathrm{Cs}^{+}$compared to $\mathrm{Na}^{+}$and not its affinity for clay surfaces.

PACS numbers:
\end{abstract}

Keywords: clay, ionic exchange, thermodynamics, molecular simulation, microcalorimetry 


\section{INTRODUCTION}

Clays are layered aluminosilicate minerals found in large amount in the Earth's upper crust and are involved in many industrial and environmental processes. In particular, their retention properties have made clayey materials possible candidates to act as barriers for the disposal of toxic and radioactive waste. In smectite clays such as montmorillonite, the layers bear a permanent negative charge compensated by counterions located between them (interlayer space). These counterions are the origin of two interesting features : clay swelling and ionic exchange. The former refers to the entrance of water into the interlayer, while the later involves the replacement of natural counterions like $\mathrm{Na}^{+}$in the clay interlayer by other ions initially in the aqueous solution in contact with the mineral, and the concomitant release of $\mathrm{Na}^{+}$in the solution [1-8]. The swelling properties of clays have been studied extensively, both experimentally [9-16] and theoretically [17-27] and are now relatively well understood. In the context of the geological disposal of nuclear waste, it is particularly important to understand the retention of radioactive traces by the geological medium. Although several mechanisms are at play for the retention of ions by clays [28-30], ionic exchange is predominant for some of them, in particular $\mathrm{Cs}^{+}$, a long-lived radioisotope of which $\left({ }^{137} \mathrm{Cs}^{+}\right)$is expected to be one of the main contributors to the long term radioactivity [31]. In order to predict how cationic radioactive species are retained by clays, it is therefore important to have a good understanding of the thermodynamic properties of ionic exchange, i.e. the associated free energy, which controls the thermodynamic constant of the reaction, and enthalpy, which controls its variations with temperature via the van't Hoff equation. This is particularly important in the context of geological disposal of nuclear waste, since the radioactive parcels will heat the surrounding medium.

Several measurements of the equilibrium constants for ionic exchange in clay minerals, particularly in those of the montmorillonite type, have been reported [3-8], but the enthalpies of exchange were much less frequently determined $[1,2,6,7,32]$. One difficulty for rigorously defining thermodynamic quantities of reaction in the case of ionic exchange is that the water content in the interlayer may vary between the initial and final states. This is one of the reasons why the exchange enthalpy and free energy depend on the degree of exchange $\alpha$. It is therefore important to distinguish the exchange of traces $\left(\alpha \rightarrow 0\right.$ for traces of $\mathrm{Cs}^{+}$in a Na-clay) from the complete exchange $(\alpha \rightarrow 1$ for the exchange from homoionic Na-clay 
to homoionic Cs-clay) [32]. Furthermore, the ionic selectivity, from which ionic exchange free energies are computed, is measured for clay suspensions. In many practical situations, however, clays constitute a solid phase (with a fixed, low water content) and the ions (e.g. traces of radioactive ions in the underground) are dissolved in the aqueous phase in contact with this solid. One thus needs to consider the water content in the clay phase when discussing the thermodynamics of ionic exchange.

According to the current paradigm for ionic exchange in clays, specific interactions between the ions and the clay surface, or the hydration properties of ions in the clay interlayer, are the driving force for the ionic exchange. The origin of clay selectivity for alkaline ions according to the series $\mathrm{Cs}^{+}>\mathrm{Rb}^{+}>\mathrm{K}^{+}>\mathrm{Na}^{+}>\mathrm{Li}^{+}$has been explained by a number of arguments : the size of hydrated cations, compared to the interlayer spacing [33-35], their ability to lose a water molecule at the clay surface, thus forming a stronger inner-sphere complex [6,36], their hydration state in the interlayer [37] or their polarizability that influences the formation of surface complexes [35, 38, 39]. These microscopic features are then thought to weight in favour of the interactions of clay interlayers with larger alkaline cations. However, considering only the clay phase to infer properties of the ionic exchange can lead to erroneous conclusions. This fact was emphasized by Teppen and Miller, who analysed the free energy of ionic exchange of small alkaline ions for larger ones, using molecular simulation supported by the analysis of experimental data from the literature [40]. They showed that the contribution of the clay phase to the ionic exchange free energy is in fact very unfavourable $(\Delta G \gg 0)$ and that it is the hydration free energy difference, i.e. the contribution of the water phase, that leads to an overall exchange of small alkaline ions for larger ones (e.g. $\mathrm{Na}^{+}$for $\mathrm{Cs}^{+}$).

In the present paper, we investigate (a) the influence of the interlayer water content on the exchange enthalpy and (b) the origin of this enthalpy. Both issues are addressed by considering thermodynamic cycles involving exchange enthalpies in solution, immersion enthalpies of clays, and hydration enthalpies of cations. This strategy is particularly efficient to investigate the ionic exchange process, because all these quantities are directly measurable by microcalorimetry experiments. It should be noted that, contrary to immersion enthalpies, immersion free energies are not measurable directly, which means that a similar analysis of the exchange free energy for well-defined interlayer water contents would be difficult, if possible at all. We analyse the contribution of the clay phase (at well defined interlayer 
water contents) and that of the aqueous solution to the ionic exchange enthalpy. We support our experimental study by directly computing by molecular simulation the contribution of the clay phase to the exchange enthalpy. From this combined experimental and theoretical analysis we demonstrate that replacing $\mathrm{Na}^{+}$ions by $\mathrm{Cs}^{+}$in the interlayer of montmorillonite clays is an endothermic process, and that the overall exchange is exothermic only because it is dominated by the exothermic replacement of $\mathrm{Cs}^{+}$by $\mathrm{Na}^{+}$in the aqueous phase.

The paper is organized as follows. We first introduce the thermodynamic quantities associated with the ionic exchange and the thermodynamic cycles allowing us (a) to determine well-defined standard reaction enthalpies and (b) to characterize the relative contribution of the clay and aqueous phase to the overall exchange thermodynamics. We subsequently describe the experimental systems and methods, and present the results of microcalorimetry measurements. Then the molecular simulation systems and methods are presented. After validating their choice by studying the free energy associated with the exchange of traces, we present the simulation results on the complete exchange enthalpy. Finally, we discuss the experimental and simulation results and demonstrate how their combination supports our conclusions.

\section{IONIC EXCHANGE THERMODYNAMICS}

\subsection{Ionic exchange reaction}

The reaction of ionic exchange between clays at a given water content and ions in a dilute aqueous solution can be written as:

$$
\overline{\mathrm{Na}^{+}} n \mathrm{H}_{2} \mathrm{O}+\mathrm{Cs}_{(a q)}^{+} \quad \longrightarrow \quad \overline{\mathrm{Cs}^{+}} n \mathrm{H}_{2} \mathrm{O}+\mathrm{Na}_{(a q)}^{+}
$$

As the reaction proceeds, the degree of exchange $\alpha$ varies from 0 (exchange of traces of $\mathrm{Cs}^{+}$ in a Na-clay) to 1 (complete exchange from homoionic Na-clay to homoionic Cs-clay). In order to define reaction quantities, it is necessary to carefully define the initial and final reference states and ensure that mass (including water) is conserved during the reaction. The standard reaction enthalpy, for the complete exchange from MNa to MCs with the same water content ( $n$ water molecules per cations), is $\Delta_{r} H^{0}(n)$. Table I summarizes the different thermodynamic quantities and the notations used in this paper. All previous experimental studies of the thermodynamics of ionic exchange were conducted on clays dispersed in water, 
i.e. in equilibrium with an aqueous phase, but in which the interlayer water content is unknown and might even differ between the initial and final states. Here we study $\Delta_{r} H^{0}(n)$ (a) by combining thermodynamic data on ionic exchange for clays dispersed in solution and on immersion of clays with a known water content, as measured by microcalorimetry, and (b) by computing directly enthalpy differences using molecular simulation.

\subsection{Thermodynamic states and water content}

In order to define reaction quantities, it is necessary that the number of water molecules remains constant. Experimentally, however, the clay water content depends both on the nature of the counterion and on the water chemical potential. Well-defined water contents can be obtained by equilibrating dry clay powders with atmospheres of controlled relative humidity $[9,10]$. It is well known that only a few hydration states are thermodynamically stable : they correspond approximately to 6-7 $\mathrm{H}_{2} \mathrm{O}$ per cation (monolayer), both for MNaand MCs, and 12-14 $\mathrm{H}_{2} \mathrm{O}$ per cation (bilayer), only for MNa. Intermediate water contents correspond to mixtures of mono- and bilayers. For very low relative humidities, a lower water content can be obtained, down to $1.4 \mathrm{H}_{2} \mathrm{O}$ per cation for $\mathrm{MNa}$, and $1.0 \mathrm{H}_{2} \mathrm{O}$ per cation for MCs.

Moreover, the exchange enthalpy can be measured directly only in the case of a clay suspension, i.e. in equilibrium with an aqueous solution, symbolized by the $\infty \mathrm{H}_{2} \mathrm{O}$ superscript in the cycle below. In that case, the water content inside the clay phase is unknown and the measured $\Delta H^{\circ}$, expressed per mole of cation, is not a reaction quantity because the water content might be different for MNa and MCs. In order to obtain experimentally the reaction enthalpy $\Delta_{r} H^{0}(n)$ corresponding to $n \mathrm{H}_{2} \mathrm{O}$ per cation, we introduce the following thermodynamic cycle:

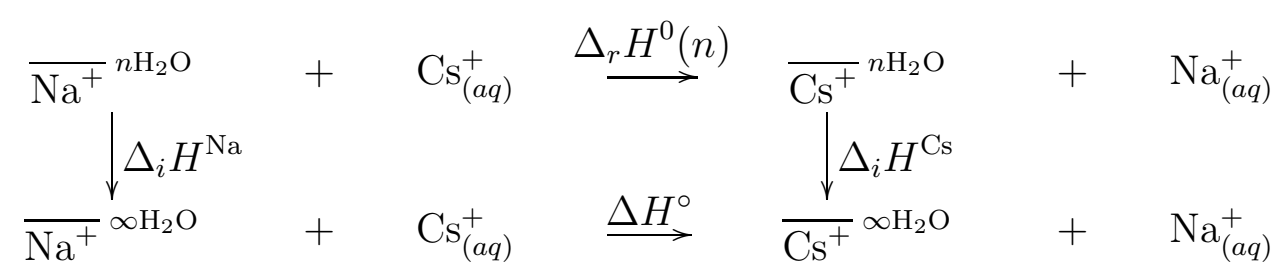

where $\Delta_{i} H$ is the enthalpy released upon immersion of a clay with a known water content into liquid water. We measure this immersion enthalpy by microcalorimetry as a function of counterion and water content, and combine it with $\Delta H^{\circ}$ for the exchange in solution to 
obtain the ionic exchange enthalpy for well-defined water content as:

$$
\Delta_{r} H^{0}(n)=\Delta H^{\circ}-\Delta \Delta_{i} H
$$

where $\Delta \Delta_{i} H=\Delta_{i} H^{\mathrm{Cs}}-\Delta_{i} H^{\mathrm{Na}}$. We will explain in section 3 how we determine experimentally the immersion enthalpies for clays with $n$ water molecule per cations.

\subsection{Origin of the ionic exchange enthalpy}

According to the current paradigm for ionic exchange in clays, specific interactions between the ions and the clay surface or/and the hydration properties of ions in the clay interlayer are the driving force for the ionic exchange. Only a single analysis of the ionic exchange free energy by Teppen and Miller [40] emphasized the importance of also considering the contribution of the aqueous phase. There are indeed two contributions to the standard reaction enthalpy: on the one hand the ion-clay and ion-water interactions in the clay phase, on the other hand the ion-water interactions in the aqueous phase. This can be understood in terms of the following thermodynamic cycle, in which we introduce the exchange reaction with ions in the gas phase, where there are no interactions, used as a reference state.



This thermodynamic cycle involves the hydration enthalpy of both cations $\Delta_{h} H^{\mathrm{Na}}$ and $\Delta_{h} H^{\mathrm{Cs}}$, the standard ionic exchange reaction with the gas phase $\Delta_{r} H_{g}^{0}(n)$ and that with the aqueous phase $\Delta_{r} H^{0}(n)$. From this cycle we deduce the relation :

$$
\Delta_{r} H_{g}^{0}(n)=\Delta_{r} H^{0}(n)+\Delta \Delta_{h} H
$$

where $\Delta \Delta_{h} H=\Delta_{h} H^{\mathrm{Cs}}-\Delta_{h} H^{\mathrm{Na}}$. After determining $\Delta_{r} H^{0}(n)$ by microcalorimetry (see section 3), we will be in a position to estimate the clay contribution $\Delta_{r} H_{g}^{0}(n)$ to the enthalpy for the exchange of ions between clays of fixed water content, and thus to investigate this quantity for well-defined reference states. In parallel to this indirect experimental determination we will also compute directly $\Delta_{r} H_{g}^{0}(n)$ using molecular simulation (see section 4 ). 


\begin{tabular}{|l|l|}
\hline Notation & Definition \\
\hline \hline$H, F, G$ & Enthalpy, Helmholtz free energy, Gibbs free energy \\
$\Delta_{h} X$ & Hydration quantity $(X=H, F, G)$ for the introduction of a gas into liquid water \\
$\Delta_{i} X$ & Immersion quantity for the introduction of a solid phase into liquid water \\
$\Delta_{r} X^{\circ}$ & Standard reaction quantity \\
$\Delta_{X^{\circ}}$ & Exchange quantity for clay dispersed in solution, per mole of cation \\
$\Delta_{r} X^{\circ}(n)$ & Standard reaction quantity for the exchange of ions between clay with $n \mathrm{H}_{2} \mathrm{O}$ \\
$\Delta_{r} X_{g}^{\circ}(n)$ & $\begin{array}{l}\text { and the aqueous phase } \\
\text { Standard reaction quantity for the exchange of ions between clay with } n \mathrm{H}_{2} \mathrm{O} \\
\text { and the gas phase }\end{array}$ \\
\hline
\end{tabular}

TABLE I: Notations used for thermodynamic quantities.

\section{MICROCALORIMETRY EXPERIMENTS}

\subsection{Materials}

Homoionic Na- and Cs-montmorillonite clays (MNa and MCs, respectively) were prepared from ANDRA's reference Wyoming bentonite (MX80). The bentonite was first purified : after removal of impurities (quartz, feldspars, etc.) by sedimentation of a suspension in deionized water, the clay solution was centrifuged several times and the upper part containing particles $<2 \mu \mathrm{m}$ was collected. Then homoionic MNa was obtained by ion-exchange : the clay was suspended in $1 \mathrm{M}$ aqueous $\mathrm{NaCl}$ and dialyzed to remove the excess salt. Conductivity measurements were used to check the presence or absence of salt in the dialyte. This ion-exchange procedure was repeated twice. The absence of $\mathrm{Cl}^{-}$ions in the dialyte was confirmed by the $\mathrm{AgNO}_{3}$ test. The homoionic Cs-montmorillonite was obtained from the Na-montmorillonite following the same procedure with $1 \mathrm{M}$ aqueous $\mathrm{CsCl}$. The purified clays were then dried under vacuum at $100^{\circ} \mathrm{C}$, and placed in a silicagel desiccator where their mass remained constant. Their purity was checked by X-ray diffraction. Dried samples were then placed in a desiccator containing saturated saline solutions, which control the relative humidity ( $\mathrm{RH})$. With saturated solutions of $\mathrm{K}_{2} \mathrm{CO}_{3}$ and $\mathrm{KCl}$, the relative humidity is of $43 \%$ and $85 \%$, respectively. The mass of the samples is stabilized within a few days. 


\subsection{Microcalorimetric measurements}

Immersion enthalpies of MNa and MCs at different relative humidities have been measured at $298 \mathrm{~K}$ using the Microsolution 2265 unit of the isothermal microcalorimeter TAM 2277 (Thermometric - TA Instruments). Samples of 5 to $25 \mathrm{mg}$ are placed in three microcartridges which fit tightly in the measurement cell. The immersion process is started by the successive fall of the cartridges into $15 \mathrm{~mL}$ of water placed in a $20 \mathrm{~mL}$ vessel stirred by a propeller at $60 \mathrm{rpm}$. Figure 1 illustrates the typical shape of the resulting thermogram. The return to baseline after immersion of a clay sample takes a time reflecting the time constant of the microcalorimeter, of the order of 10 minutes, which is longer than the hydration process itself. Immersion enthalpies (in $\mathrm{mJ} / \mathrm{mg}$ ) are computed by integrating the thermogram peaks using the Digitam software (Thermometric). They are given in $\mathrm{kJ} / \mathrm{mol}$ of cation in table II. Three to six immersions were carried out for each montmorillonite sample, with given counterion and hydration state. The reproducibility of the measurements is very good for $\mathrm{RH}=43 \%$ and $85 \%$, however there is more variability for the samples prepared at $\mathrm{RH}=0 \%$. This is due to the fact that the immersion enthalpy varies significantly with the water content when the latter is small $[9,10]$, and that the water content of the driest samples can increase during the preparation of the microcalorimetry experiment.

\begin{tabular}{|c|c|c|}
\hline $\operatorname{HR}(\%)$ & $\Delta_{i} H^{\mathrm{Na}}\left(\mathrm{kJ} . \mathrm{mol}^{-1}\right)$ & $\Delta_{i} H^{\mathrm{Cs}}\left(\mathrm{kJ} . \mathrm{mol}^{-1}\right)$ \\
\hline \hline 0 & $-37 \pm 4$ & $-21 \pm 4$ \\
43 & $-25 \pm 1$ & $-5 \pm 1$ \\
85 & $-8 \pm 1$ & $-4 \pm 1$ \\
\hline
\end{tabular}

TABLE II: Experimental immersion enthalpies for Na- and Cs-montmorillonite, as a function of the relative humidity $(\mathrm{RH})$. Results are given per mole of cation.

Exchange enthalpies for clays dispersed in water at $298 \mathrm{~K}$ were obtained as explained in ref. [32]. The exchange enthalpy depends on the degree of exchange $\alpha$, with values ranging from $-15 \pm 0.5 \mathrm{~kJ} / \mathrm{mol}$ of cation for $\alpha \rightarrow 0$ (which corresponds to traces of $\mathrm{Cs}^{+}$in a MNa) to $-4.1 \pm 0.2 \mathrm{~kJ} / \mathrm{mol}$ for $\alpha \rightarrow 1$. The standard reaction enthalpy is obtained by integrating these values between $\alpha=0$ and 1 , with the result $\Delta H^{0}=-7.5 \pm 1.0 \mathrm{~kJ} / \mathrm{mol}$. The standard state corresponds to clay in solution at infinite dilution, where the interlayer in equilibrium 
with the solution has an unknown water content.

\subsection{Immersion enthalpy as a function of water content}

We have shown using the thermodynamic cycle (2) how the exchange enthalpy $\Delta_{r} H^{0}(n)$ can be deduced from the exchange enthalpy in solution $\Delta H^{\circ}$ and the immersion enthalpies $\Delta_{i} H^{\mathrm{Na}}$ and $\Delta_{i} H^{\mathrm{Cs}}$ via Eq. 3. These immersion enthalpies must be determined as a function of the water content $n$. As mentioned in section 2 , the experimental hydration states correspond to clays in equilibrium with vapor at a given relative humidity. Only a few hydration states are thermodynamically stable and most conditions correspond to mixtures of these states. Table III reports the proportion of each state as a function of the relative humidity, as estimated by Bérend et al. for Na- and Cs-Wyoming montmorillonite (Clay Spur 26 from Ward's Natural Science) [10].

\begin{tabular}{|c|c|c|c|c|c|c|}
\hline \multirow{2}{*}{ RH (\%) } & \multicolumn{3}{|c|}{ Na } & \multicolumn{3}{c|}{ Cs } \\
\cline { 2 - 7 } & dry (\%) & mono (\%) & bi (\%) & dry (\%) & mono (\%) & bi (\%) \\
\hline \hline 0 & 100 & 0 & 0 & 100 & 0 & 0 \\
43 & 50 & 50 & 0 & 40 & 60 & 0 \\
85 & 0 & 20 & 80 & 20 & 80 & 0 \\
\hline
\end{tabular}

TABLE III: Proportion of hydration states: dry (1-2 water molecules per cation), monolayer (6-7) and bilayer (12-14) as a function of the relative humidity (RH) of the atmosphere in equilibrium with the clay. These values are estimations from Bérend et

$$
\text { al. [10]. }
$$

Although obtained for samples of a different montmorillonite, prepared under slightly different conditions, these results can be used together with table II to estimate the value of the immersion enthalpy $\Delta_{i} H(n)$ for $n=1$ and 6 . The values for $\mathrm{RH}=0 \%$ correspond to the values for $n=1$, thus $\Delta_{i} H^{\mathrm{Na}}(n=1)=-37 \pm 4 \mathrm{~kJ} / \mathrm{mol}$ and $\Delta_{i} H^{\mathrm{Cs}}(n=1)=-21 \pm 4 \mathrm{~kJ} / \mathrm{mol}$. Then we can deduce the values for $n=6$ (monolayer), since we have $\Delta_{i} H^{\mathrm{Na}}(R H=43 \%)=$ $0.5 \Delta_{i} H^{\mathrm{Na}}(n=1)+0.5 \Delta_{i} H^{\mathrm{Na}}(n=6)$. This yields $\Delta_{i} H^{\mathrm{Na}}(n=6)=-13 \pm 6 \mathrm{~kJ} / \mathrm{mol}$, and a similar calculation gives $\Delta_{i} H^{\mathrm{Cs}}(n=6)=+6 \pm 4 \mathrm{~kJ} / \mathrm{mol}$. Note that the main contribution to the uncertainty comes from the estimation of the water content, and not 
from the microcalorimetric measurements of immersion enthalpies. The positive sign for the immersion of MCs in the monohydrated state is consistent with the fact that MCs does not swell. This also holds for K-montmorillonite and a positive sign was found in that case by molecular simulation results [23].

\subsection{Complete exchange enthalpy}

From the immersion enthalpies for $n=1$ and 6 , which are stable states for both counterions, and the exchange enthalpy in solution $\Delta H^{\circ}$ we now obtain the standard reaction enthalpy using Eq. (3). This yields $\Delta_{r} H^{0}(1)=-7.5-[-21+37]=-23.5 \pm 9 \mathrm{~kJ} / \mathrm{mol}$ and $\Delta_{r} H^{0}(6)=-7.5-[6-(-13)]=-26.5 \pm 11 \mathrm{~kJ} / \mathrm{mol}$. The main contribution to the uncertainty comes again from the estimate of the water content for a given relative humidity, and not from the measurements of enthalpies by microcalorimetry. We repeated the same analysis using the experimental immersion enthalpies of Bérend et al. [9]. In that case Eq. (3) yields $-39.5 \pm 7 \mathrm{~kJ} / \mathrm{mol}$ for $n=1$, and $-16.5 \pm 15 \mathrm{~kJ} / \mathrm{mol}$ for $n=6$. Despite the relatively large uncertainties, both sets of data lead to consistent results : They clearly indicate that the ionic exchange is exothermic. Note that the ionic exchange enthalpy for $n=1$ and 6 are more negative than the value obtained in solution $\left(\Delta H^{\circ}=-7.5 \mathrm{~kJ} / \mathrm{mol}\right.$ of cation). This underlines the need to consider carefully the water content in clays when analysing thermodynamic quantities.

\section{MOLECULAR SIMULATION}

Molecular simulation allows to compute the energy and enthalpy of a system from the interactions between the atoms, and thus gives access to differences in thermodynamic quantities that are not easily accessible by direct measurement. For example, the reaction energy $\Delta_{r} U_{g}^{0}(n)$ of the ion-exchange reaction between a) the clay at a well-defined water content of $n$ water molecules per cations, and b) ions in the gas phase (which can be used as a reference

state for the ions) is obtained by substracting the energy of the MNa system to that of the MCs system (there are no interactions for isolated ions in the gas phase). Similarly, the hydration enthalpy difference $\Delta \Delta_{h} H$ for $\mathrm{Na}^{+}$and $\mathrm{Cs}^{+}$is obtained by computing the enthalpy of the ions in water (again, there is no contribution of the gas phase). Ionic exchange free 
energies can also be computed using the thermodynamic integration method [41, 42], as will be explained in section 44.2 .

\subsection{System}

The simulated clay system has the idealized composition

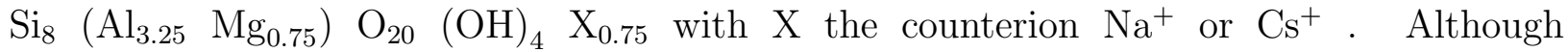
no experiments for $\mathrm{K}^{+}$montmorillonite were undertaken in the present study, we also performed molecular simulations for this counterion. The simulation box of lateral dimensions $20.72 \times 17.94 \AA^{2}$ contains two clay layers, both with $4 \times 2$ unit cells and a thickness of $6.54 \AA$ (distance between surface oxygen atoms from the same layer). Their atomic structure was taken from X-ray diffraction measurements [43]. The interlayer space contains the counterions and $n$ water molecules per cation. Periodic boundary conditions in all directions are used so that the simulation box corresponds to infinite clay layers (no edges). A snapshot of the simulation box is represented in Fig. 2.

Interactions between atoms are modelled using pairwise potentials :

$$
\mathcal{V}_{i j}=\frac{q_{i} q_{j}}{4 \pi \epsilon_{0} r_{i j}}+4 \varepsilon_{i j}\left[\left(\frac{\sigma_{i j}}{r_{i j}}\right)^{12}-\left(\frac{\sigma_{i j}}{r_{i j}}\right)^{6}\right]
$$

with $q_{i}$ the (partial) charge of atom $i$, and the pair Lennard-Jones (LJ) parameters $\varepsilon_{i j}$ and $\sigma_{i j}$ are calculated from the individual $\varepsilon_{i i}$ and $\sigma_{i i}$ using Lorentz-Berthelot mixing rules. $\mathcal{V}_{i j}$ contains an electrostatic (Coulomb) term, and a 6-12 Lennard-Jones term. This force field is completely determined by the atomic parameters $\left\{q_{i}, \varepsilon_{i i}, \sigma_{i i}\right\}$. Water molecules were described using the rigid SPC/E model [44]. The ions LJ parameters are taken from Åqvist [45]. They were developped to reproduce the experimental hydration energy when used in combination with the SPC water model (SPC/E is an extension of this model). The clay layers are treated as rigid bodies. The partial charges and LJ parameters for clay atoms are the one from Smith [46]. In this force field the LJ parameters for surface oxygen atoms are identical to that of SPC/E water oxygens. All partial charges and individual Lennard-Jones parameters are summarized in Electronic Annex 1. Long-range electrostatic interactions were computed using Ewald summation, and a cut-off of $8 \AA$ was used for the short-range LJ interactions. 


\subsection{Methods}

We performed Monte-Carlo simulations in the NPT ensemble, i.e. at fixed number of particles, pressure $P=1$ bar, and temperature $T=298 \mathrm{~K}$. Thermodynamic quantities such as energy and enthalpy were computed by averaging their mechanical expressions $U=$ $\sum_{i<j} \mathcal{V}_{i j}$ and $H=U+P V$ over microscopic configurations sampled from the NPT ensemble. For the clay systems we did not allow the rotation of the layers relative to each other, but only translational moves in the direction perpendicular to the surfaces, and in both directions along the surfaces. Only the pressure in the direction perpendicular to the clay surfaces is imposed $\left(N P_{z} T\right.$ ensemble). This is necessary to preserve the symmetry of the clay particle. We performed simulations for both $\mathrm{Na}^{+}$and $\mathrm{Cs}^{+}$counterions, and water contents of 1 (dry state), 6 (monolayer) and 12 (bilayer) water molecules per cations. Ions and solvent molecules are inserted randomly in the interlayers, and the system is first equilibrated at $1000 \mathrm{~K}$ for $10^{5}$ steps. The temperature is then gradually decreased to $298 \mathrm{~K}$. After $10^{7}$ equilibration steps at this temperature, sampling of the energy and enthalpy is achieved for $510^{7}$ to $10^{8}$ steps. For the lowest hydration states, simulated annealings (i.e. repeating the above procedure via high temperatures) are used to ensure that the simulation correctly samples the whole phase space. The equilibrium interlayer distances obtained by $N P_{z} T$ simulation are $\approx 10.8,12.3$, and $15.3 \AA$ for $\mathrm{Na}^{+}$with $n=1,6$ and 12 , respectively, and 12.2 and $12.9 \AA$ for $\mathrm{Cs}^{+}$with $n=1$ and 6 , respectively. These results are in good agreement with X-ray diffraction data, except for the dryest states for which smaller distances have been reported (see e.g. [10, 12]). In that case, even a single water molecule leads to a larger distance than for a completely dry state.

Simulations of aqueous ions consisted of a single ion and 252 water molecules in a cubic box. The equilibrium size of the box corresponds to a concentration of about $0.2 \mathrm{M}$. The fact that the simulation box is charged (there is no anion) is not a problem : it has been shown that it is equivalent to the same system with a uniform neutralizing background, and that there is only a shift in the electrostatic energy [47]. For a cubic box of size $L$, $\Delta E=-\alpha Q^{2} / 8 \pi \epsilon_{0} L$, with $Q$ the total charge of the box and $\alpha$ a (Madelung) constant. Thus the shift is identical for the simulations with $\mathrm{Na}^{+}$or $\mathrm{Cs}^{+}$and compensates when considering differences in energy (or enthalpy).

In addition to Monte-Carlo simulations in the NPT ensemble, we also performed molec- 
ular dynamics simulations of the same systems in the NVT ensemble (at the equilibrium volume determined by the NPT simulations), using a modified version of the DLPOLY software package. These simulations were used to compute Helmholtz free energy differences by the thermodynamic integration technique [41, 42]. It consists in gradually turning one type of ion into another using a coupling parameter $\lambda \in[0,1]$ which interpolates between the Hamiltonian $\mathcal{V}(\lambda=0)=\mathcal{V}^{\mathrm{Na}}$ of the system with $\mathrm{Na}^{+}$and that $\mathcal{V}(\lambda=1)=\mathcal{V}^{\mathrm{Cs}}$ with $\mathrm{Cs}^{+}$. Then the Helmholtz free energy difference between the two states is :

$$
\Delta F^{\mathrm{Na} \rightarrow \mathrm{Cs}}=\int_{0}^{1}\left\langle\frac{\partial \mathcal{V}}{\partial \lambda}\right\rangle_{\lambda} \mathrm{d} \lambda
$$

The term in the integral is an ensemble average for a system at a fixed, intermediate value of $\lambda$. We have chosen a thermodynamic path in which all the pair LJ parameters vary linearly between that of $\mathrm{Na}$ and that of $\mathrm{Cs}$ :

$$
\begin{gathered}
\varepsilon_{\lambda, j}=\varepsilon_{\mathrm{Na}, j}+\lambda\left(\varepsilon_{\mathrm{Cs}, j}-\varepsilon_{\mathrm{Na}, j}\right) \\
\sigma_{\lambda, j}=\sigma_{\mathrm{Na}, j}+\lambda\left(\sigma_{\mathrm{Cs}, j}-\sigma_{\mathrm{Na}, j}\right)
\end{gathered}
$$

where $j$ corresponds to any other atom, and where the extreme pair parameters $\varepsilon_{\mathrm{Na}, j}, \varepsilon_{\mathrm{Cs}, j}$, $\sigma_{\mathrm{Na}, j}$, and $\sigma_{\mathrm{Cs}, j}$ are obtained from the Lorentz-Berthelot rules. We considered thermodynamic paths in which a single ion is changed from $\mathrm{Na}^{+}$to $\mathrm{Cs}^{+}, \mathrm{Na}^{+}$to $\mathrm{K}^{+}$, and $\mathrm{K}^{+}$to $\mathrm{Cs}^{+}$, both in pure water and in clay at a water content of 6 water molecules per cation, corresponding to a water monolayer. For each thermodynamic integration we performed a set of 11 simulations at different $\lambda$ corresponding to a step $\Delta \lambda=0.1$, in which we sampled $\langle\partial \mathcal{V} / \partial \lambda\rangle$ for at least 100 ps. Fig. 3 shows the variation of the average $\langle\partial \mathcal{V} / \partial \lambda\rangle$ as a function of $\lambda$ for the thermodynamic integration from $\mathrm{Na}^{+}$to $\mathrm{Cs}^{+}$in aqueous solution (solid line) and in clay with $n_{H 2 O}=6$ water molecules per cation (dashed line). Numerical integration between $\lambda=0$ and 1 yields the difference in hydration free energy $\Delta_{h} F$ and the enthalpy for the exchange of ions between clay and the gas phase $\Delta_{r} F^{0}(6)$, respectively.

\subsection{Free energy for the exchange of traces}

We first present the results of the thermodynamic integration approach, and use them to validate our choice of parameters to describe the energetic properties of clay, ions and water. In the aqueous ions simulations, the resulting free energy difference corresponds to 
the hydration free energy difference $\Delta \Delta_{h} F$ for $\mathrm{Na}^{+}, \mathrm{K}^{+}$and $\mathrm{Cs}^{+}$. The results are reported in table IV.

\begin{tabular}{|c|c|c|c|}
\hline$\Delta \Delta_{h} F\left(\mathrm{~kJ}^{\mathrm{mol}}{ }^{-1}\right)$ & Simulation & Burgess & Marcus \\
\hline \hline $\mathrm{Na}^{+} \rightarrow \mathrm{Cs}^{+}$ & $+124 \pm 2$ & +127 & +117 \\
$\mathrm{Na}^{+} \rightarrow \mathrm{K}^{+}$ & $+72 \pm 2$ & +74 & +72 \\
$\mathrm{~K}^{+} \rightarrow \mathrm{Cs}^{+}$ & $+53 \pm 2$ & +53 & +45 \\
\hline
\end{tabular}

TABLE IV: Hydration free energy difference as obtained by thermodynamic integration. Our simulation results are compared to the values given in the thermodynamic databases compiled by Burgess [48] and Marcus [49].

We first notice that there is a good thermodynamic consistency between the results, i.e. that $\Delta \Delta_{h} F(\mathrm{Na} \rightarrow \mathrm{Cs})=\Delta \Delta_{h} F(\mathrm{Na} \rightarrow \mathrm{K})+\Delta_{h} F(\mathrm{~K} \rightarrow \mathrm{Cs})$. Furthermore, the simulation results are in good agreement with the experimental data for $\Delta \Delta_{h} G \sim \Delta \Delta_{h} F$ (the difference $P \Delta V$ between these two is only a few $\mathrm{kJ} / \mathrm{mol}$ ) reported in [50] $:+117,+72$ and $+45 \mathrm{~kJ} / \mathrm{mol}$, and even better with the values of ref. [48] : $+127,+74$ and $+53 \mathrm{~kJ} / \mathrm{mol}$. The excellent agreement with the latter is not surprizing, since the Lennard-Jones parameters for the cations were calibrated by Åqist to reproduce these values when combined to the SPC water model [45], a variant of which (SPC/E) is used in this study. Therefore the force field used for our simulations reasonably represents the interactions between ions and water.

\begin{tabular}{|c|c|}
\hline Transformation & $\Delta_{r} F_{g}^{0}(6)\left(\mathrm{kJ} . \mathrm{mol}^{-1}\right)$ \\
\hline \hline $\mathrm{Na}^{+} \rightarrow \mathrm{Cs}^{+}$ & $+120 \pm 2$ \\
$\mathrm{Na}^{+} \rightarrow \mathrm{K}^{+}$ & $+68 \pm 2$ \\
$\mathrm{~K}^{+} \rightarrow \mathrm{Cs}^{+}$ & $+51 \pm 2$ \\
\hline
\end{tabular}

TABLE V: Free energy difference obtained by thermodynamic integration for ions in clay with 6 water molecules per cation.

The results for the thermodynamic integration for the exchange of a single ion in a MNa with 6 water molecules per counterions are reported in table V. We observe again a good thermodynamic consistency between the results. In addition, all values are positive and 
relatively large, meaning that the replacement of an alkaline ion by a larger one in a $\mathrm{MNa}$ is clearly thermodynamically unfavourable. More precisely, the value of $\Delta_{r} F_{g}^{0}(6)$ corresponds to the exchange of ions between a $\mathrm{MNa}$ and the gas phase (see below). The value for the $\mathrm{Na}^{+} \rightarrow \mathrm{Cs}^{+}$exchange is in good agreement with the conclusion drawn by Teppen and Miller [40] from experimental data $(+117 \mathrm{~kJ} / \mathrm{mol})$, and the one for the $\mathrm{K}^{+} \rightarrow \mathrm{Cs}^{+}$exchange is in good agreement with both their simulation results $(+47 \mathrm{~kJ} / \mathrm{mol})$ and their conclusions drawn from experimental data $(+54 \mathrm{~kJ} / \mathrm{mol})$. Since their simulation results were obtained using a different force field to represent ions and clay atoms, and a slightly different method to compute the free energy difference (free energy perturbation, see e.g. [42]), the similar conclusion to which we arrive, namely that the clay phase contributes unfavourably to the ionic exchange free energy, seems to be very robust. We can also conclude that the force field that we use gives a reasonable description of the thermodynamic properties of clay interlayers.

In the above-mentioned study, the authors considered the complete exchange from a Kclay to a Cs-clay, whereas here we consider the exchange of ionic traces in a MNa. This suggests that for a given water content, the short-range ion-clay and ion-water interactions are relatively independent of the nature of the other ions in the interlayer. Such an observation has already been made in simulations of $\mathrm{Cs}^{+}$in bihydrated Na-montmorillonite [51]: the structure of the inner-sphere Cs-surface complex was very similar to the one observed in monohydrated Cs-montmorillonite. It is therefore not surprising that energetic properties for the exchange of traces of $\mathrm{Cs}^{+}$in $\mathrm{MNa}$ show the same behaviour as for the complete exchange from homoionic MNa to homoionic MCs.

\subsection{Complete exchange enthalpy}

The enthalpy for the complete $\mathrm{Na}^{+} \rightarrow \mathrm{Cs}^{+}$exchange is reported in table VI, as a function of the number of water molecules per cations: $n_{H 2 O}=1$ corresponds to the dry state, 6 to the monolayer state, and 12 to the bilayer state. Note that the latter is not experimentally stable in the Cs case [10]. As for the free energy, $\Delta_{r} H_{g}^{0}(n)$ corresponds to the exchange of ions between clay and the gas phase. These values are all positive and large. Furthermore, they increase as the water content increases. All these observations will be discussed and compared to experimental results in the next section. 


\begin{tabular}{|c|c|}
\hline$n_{\mathrm{H} 2 \mathrm{O}}$ & $\Delta_{r} H_{g}^{0}\left(\mathrm{~kJ} . \mathrm{mol}^{-1}\right)$ \\
\hline 1 & $+88 \pm 5$ \\
\hline 6 & $+101 \pm 3$ \\
\hline 12 & $+113 \pm 4$ \\
\hline
\end{tabular}

TABLE VI: Clay contribution to the standard enthalpy for the exchange reaction between a Na-Clay with $n$ water molecules per cation and a Cs-Clay with $n$ water molecules per cation (see the thermodynamic cycle 4 ). The values for $n=1,6$ and 12 correspond to the dry, monolayer and bilayer states, respectively - even if the latter is not experimentally stable in the Cs case. These simulation results should be compared to the experimental ones of table VII.

\section{DISCUSSION}

\subsection{Origin of the ionic exchange enthalpy}

The results for the contribution of the clay phase to the reaction enthalpy $\Delta_{r} H_{g}^{0}(n)$, computed from Eq. (5) using the values of $\Delta_{r} H^{0}(n)$ determined in section 33.4 and the experimental value of $\Delta \Delta_{h} H=+133 \mathrm{~kJ} / \mathrm{mol}$ [49], are reported in table VII. The same analysis using the experimental immersion enthalpies of Bérend et al. [10] yields $+93.5 \pm$ $7 \mathrm{~kJ} / \mathrm{mol}$ for $n=1$, and $+116.5 \pm 15 \mathrm{~kJ} / \mathrm{mol}$ for $n=6$.

\begin{tabular}{|c|c|}
\hline$n_{\mathrm{H} 2 \mathrm{O}}$ & $\Delta_{r} H_{g}^{0}\left(\mathrm{~kJ} . \mathrm{mol}^{-1}\right)$ \\
\hline \hline 1 & $+109.5 \pm 9$ \\
6 & $+106.5 \pm 11$ \\
$\infty$ & $+125.5 \pm 1$ \\
\hline
\end{tabular}

TABLE VII: Clay contribution to the standard enthalpy for the exchange reaction between a Na-Clay with $n$ water molecules per cation and a Cs-Clay with $n$ water molecules per cation (see the thermodynamic cycle 4). Results are obtained from the experimental immersion enthalpies, enthalpy for the exchange in solution and the proportions of each hydration states. 
Experimental results therefore clearly indicate that interactions in the clay interlayer contribute unfavourably to the replacement of $\mathrm{Na}^{+}$cations by $\mathrm{Cs}^{+}$. This conclusion is further supported by the simulation results of table VI. The latter can be seen as more direct estimates of $\Delta_{r} H_{g}^{0}(n)$ since they are based on the computation of the internal energy from the microscopic interactions between atoms. The agreement between experimental and simulation results, though not quantitative for the lowest water content, is good. The uncertainty on the experimental results reported in table VII does not allow to identify the variations of $\Delta_{r} H_{g}^{0}(n)$ with water content, but the results based on the experiments of ref. [10] suggest an increase of $\Delta_{r} H_{g}^{0}(n)$ with increasing water content, as observed in simulations.

As mentioned in the introduction, the current paradigm for ionic exchange considers that its driving force is to be found in specific interactions between the ions and the clay surface, or in the hydration properties of the ions in the clay interlayer, that would be more favourable to $\mathrm{Cs}^{+}$than to $\mathrm{Na}^{+}$. However, our simulation results for the free energy clearly support the conclusion of Teppen and Miller [40] that the contribution of the clay phase to the ionic exchange free energy is in fact very unfavourable $(\Delta G \gg 0)$ and that it is the hydration free energy difference, i.e. the contribution of the water phase, that leads to an overall exchange of $\mathrm{Na}^{+}$for $\mathrm{Cs}^{+}$. The driving force for the $\mathrm{Na}^{+}$to $\mathrm{Cs}^{+}$exchange in montmorillonite is thus the "hydrophobicity" of $\mathrm{Cs}^{+}$compared to $\mathrm{Na}^{+}$, and not its affinity for the clay surfaces. The same argument also explains the selectivity observed for alkaline earth cations in the order $\mathrm{Ba}^{2+}>\mathrm{Sr}^{2+}>\mathrm{Ca}^{2+}>\mathrm{Mg}^{2+}$, as was already suggested in ref. [40] as an extension of their analysis of alkaline cations.

Both our experimental and simulation results support the conclusion that the same holds for the ionic exchange enthalpy: replacing $\mathrm{Na}^{+}$ions by $\mathrm{Cs}^{+}$in the interlayer of montmorillonite clays is an endothermic process, and the overall exchange is exothermic only because it is dominated by the exothermic replacement of $\mathrm{Cs}^{+}$by $\mathrm{Na}^{+}$in the aqueous phase. An advantage of considering enthalpies is that they are measured directly by microcalorimetry, whereas experimental free energies in solution are deduced from exchange constants, and immersion or swelling free energies are not easily measured. Therefore enthalpy lends itself better to the direct comparison between experimental and simulation results. 


\subsection{Implications for the exchange of traces}

We mentioned in the experimental section that the exchange enthalpy depends on the degree of exchange $\alpha$. In the trace regime $(\alpha \rightarrow 0)$, the value measured in solution by microcalorimetry is $-15 \pm 0.5 \mathrm{~kJ} / \mathrm{mol}[32]$. We could not obtain a direct estimate by simulation using the same approach as before, because the change in enthalpy associated with the replacement of one $\mathrm{Na}^{+}$by one $\mathrm{Cs}^{+}$in a simulation box containing only $\mathrm{Na}^{+}$counterions is small compared to the absolute value of the enthalpy in both systems, and even to the equilibrium fluctuations of the enthalpy. Therefore the uncertainty on the difference obtained by this approach is too large for the result to be meaningful. The evaluation of the free

energy did not suffer from this problem, since it was based on a quantity $\left(\left\langle\frac{\partial \mathcal{V}}{\partial \lambda}\right\rangle\right)$ involving only the exchanged ion.

However, the enthalpy for the exchange of traces can be estimated under the assumption that ions in the interlayer behave independently of the nature of the surrounding cations. This assumption is reasonable and consistent with previous molecular simulation results. In [51], it was shown that $\mathrm{Cs}^{+}$ions in a bihydrated heteroionic $\mathrm{Na} / \mathrm{Cs}$-clay adopt the same coordination mode as in the -experimentally unstable- bihydrated homoionic MCs : three surface oxygen atoms were included in the first coordination shell of the ion, as also observed on monohydrated homoionic MCs. The value of the enthalpy can then be approximated by $\Delta_{r} H^{0}(12)=\Delta_{r} H_{g}^{0}(12)-\Delta_{h} H=+113-133=-20 \pm 4 \mathrm{~kJ} / \mathrm{mol}$. Since $\Delta_{r} H_{g}^{0}(n)$ increases with the water content, the choice of the -thermodynamically stable- bilayer state provides a lower bound on the value for suspended clays (for which the larger water content is unknown). This estimate is in good agreement with the value measured by microcalorimetry.

Finally, we can compare these results to the enthalpy deduced from the measurements of clay selectivities at various temperatures using the Van't Hoff relation. Liu et al. have reported a value of $-18 \pm 2 \mathrm{~kJ} / \mathrm{mol}$ [52], and Tertre et al. $-19 \pm 5 \mathrm{~kJ} / \mathrm{mol}$ [53]. The assumptions on $\mathrm{Cs}^{+}$in $\mathrm{MNa}$ and on the water content seem therefore to be reasonable. The fact that independent routes (direct measurement or simulation on the one hand and selectivity measurements at different temperatures on the other hand) lead to the same value of the trace exchange enthalpy give further support to the above discussion. Since the ionic exchange reaction is exothermic, it is a priori unfavoured by an elevation of temperature. However the relatively low value of the exchange enthalpy implies that temperature has only 
a weak influence on the retention of $\mathrm{Cs}^{+}$by clays, as was already concluded in [32].

\section{CONCLUSION}

We have studied the origin of the ionic exchange enthalpy in montmorillonite clays using microcalorimetry measurements and molecular simulation. We have first determined the standard reaction enthalpy for well-defined interlayer water contents. We have then shown by a detailed analysis based on thermodynamic cycles that replacing $\mathrm{Na}^{+}$ions by $\mathrm{Cs}^{+}$in the interlayer of montmorillonite clays is an endothermic process, and that the overall exchange is exothermic only because it is dominated by the exothermic replacement of $\mathrm{Cs}^{+}$by $\mathrm{Na}^{+}$ in the aqueous phase. This conclusion supports the one of a recent study of the ionic exchange free energy [40] and contradicts long-held views on the role of ion-clay interactions in determining the ionic exchange thermodynamics. This calls for a paradigm shift for the driving force of this exchange: The driving force is the "hydrophobicity" of $\mathrm{Cs}^{+}$compared to $\mathrm{Na}^{+}$and not its affinity for clay surfaces. This analysis has a broader range than the case of clays, and might explain the relative homogeneity of specific effects observed in many contexts according to "Hofmeister series" [54]: One should not forget the role of the hydration energy (i.e. the contribution of the water phase), even if specific interactions with the substrates come into play. The present study does not tell us about the role of the nature of the counterions on the kinetics of the exchange. This would require to take into account clay particle edges explicitely [55]. Moreover, particle edges provide other sites for the retention of cations [28-30]. In particular, the understanding of the effect of $\mathrm{pH}$ on

the sorption of cations on these edge sites might benefit from the combined theoretical and experimental approach followed in the present paper.

\section{Acknowledgments}

B.R. acknowledges financial support of the Agence Nationale pour la Gestion des Déchets Radioactifs (ANDRA). The authors are grateful to ANDRA for providing the MX80 bentonite samples and acknowledge financial support from the GNR PARIS. The authors thank 
the reviewers for helpful suggestions.

[1] W. H. Slabaugh. Cation exchange properties of bentonite. J. Phys. Chem., 58(2):162-165, 1954 .

[2] R. M. Barrer, D. J. Ward, and L. V. C. Rees. Thermochemistry and thermodynamics of ion exchange in a crystalline exchange medium. Proc. R. Soc. London, 273(135):180-197, 1963.

[3] H. Martin and H Laudelout. Thermodynamique de l'échange des cations alcalins dans les argiles. J. Chim. Phys., 60(9):1086-1099, 1963.

[4] J. Fripiat, P. Cloos, and A. Poncelet. Comparaison entre les propriétés d'échange de la montmorillonite et d'une résine vis-à-vis des cations alcalins et alcalino-terreux I. réversibilité des processus. Bull. Soc. Chim. Fr., (1):208-221, 1964.

[5] J. Robeyns, R. van Bladel, and H. Laudelout. Thermodynamics of singly charged ion exchanges in trace regions on Camp Berteau montmorillonite. J. Soil. Sci., 22(3):336-341, 1971.

[6] A. Maes and A. Cremers. Charge density effects in ion exchange. part 2. Homovalent exchange equilibria. J. Chem. Soc. Faraday Trans., 74:1234-1241, 1978.

[7] G. J. Ewin, B. P. Erno, and L. G. Hepler. Clay chemistry - investigation of thermodynamics of ion-exchange reactions by titration calorimetry. Can. J. Chem., 59(20):2927-2933, 1981.

[8] A. Dyer, J. K. K. Chow, and I. M. Umar. The uptake of caesium and strontium radioisotopes onto clays. J. Mater. Chem., 10(12):2734-2740, 2000.

[9] J.M. Cases, I. Bérend, M. Francois, J.-P. Uriot, F. Thomas, and J.E. Poirier. Mechanism of adsorption and desorption of water vapour by homoionic montmorillonite 1 . The sodium exchanged form. Langmuir, 8:2730-2739, 1992.

[10] I. Bérend, J.M. Cases, M. Francois, J.-P. Uriot, L.J. Michot, A. Masion, and F. Thomas. Mechanism of adsorption and desorption of water vapour by homoionic montmorillonite 2 . The $\mathrm{Li}^{+}, \mathrm{Na}^{+}, \mathrm{K}^{+}$etc. exchanged forms. Clay Clay Miner., 43:324-336, 1995.

[11] L.J. Michot, F. Villiéras, M. Francois, I. Bihannic, M. Pelletier, and J.M. Cases. Water organisation at the solid-aqueous solution interface. C. R. Geosciences, 334:611-631, 2002.

[12] E. Ferrage, B. Lanson, N. Malikova, A. Plancon, B.A. Sakharov, and V.A. Drits. New insights on the distribution of interlayer water in bi-hydrated smectite from X-ray diffraction profile modeling of 001 reflections. Chem. Mater., 17(13):3499-3512, 2005. 
[13] C.A. Weiss, R.J. Kirkpatrick, and S.P. Altaner. Variations in interlayer cation sites of clay minerals as studied by ${ }^{133} \mathrm{Cs}$ mas nuclear magnetic resonance spectroscopy. Am. Mineralogist, 75:970-982, 1990 .

[14] G. Trausch, D. Canet, A. Cadène, and P. Turq. Separation of components of a ${ }^{1} \mathrm{H}$ NMR composite signal by nutation experiments under low amplitude radio-frequency fields. application to the water signal in clays. Chem. Phys. Lett., 433(1-3):228-233, 2006.

[15] E. Rinnert, C. Carteret, B. Humbert, G. Fragneto-Cusani, J.D.F. Ramsay, A. Delville, J.L. Robert, I. Bihannic, M. Pelletier, and L.J. Michot. Hydration of a synthetic clay with tetrahedral charges: A multidisciplinary experimental and numerical study. J. Phys. Chem. B, 109(49):23745-23759, 2005.

[16] F. Salles, I. Beurroies, O. Bildstein, M. Jullien, J. Raynal, R. Denoyel, and H. Van Damme. A calorimetric study of mesoscopic swelling and hydration sequence in solid na-montmorillonite. Appl. Clay Sci., 39(3-4):186-201, 2008.

[17] E.S. Boek, P.V. Coveney, and N.T. Skipper. Molecular modeling of clay hydration: a study of hysteresis loops in the swelling curves of sodium montmorillonites. Langmuir, 11(12):46294631,1995

[18] E.S. Boek, P.V. Coveney, and N.T. Skipper. Monte Carlo molecular modelling studies of hydrated Li-, Na- and K-smectites: Understanding the role of potassium as a clay swelling inhibitor. J. Am. Chem. Soc., 117(50):12608-12617, 1995.

[19] A.V.C. de Siqueira, N.T. Skipper, P.V. Coveney, and E.S. Boek. Computer simulation evidence for enthalpy driven dehydration of smectite clays at elevated pressures and temperatures. Mol. Phys., 92(1):1-6, 1997.

[20] D.A. Young and D.E. Smith. Simulations of clay mineral swelling and hydration: Dependence upon interlayer ion size and charge. J. Phys. Chem. B, 104(39):9163-9170, 2000.

[21] H.D. Whitley and D.E. Smith. Free energy, energy, and entropy of swelling in Cs-, Na-, and Sr-montmorillonite clays. J. Chem. Phys., 120(11):5387-5395, 2004.

[22] D.E. Smith, Y. Wang, A. Chaturvedi, and H.D. Whitley. Molecular simulations, of the pressure, temperature, and chemical potential dependencies of clay swelling. J. Phys. Chem. B, 110(40):20046-20054, 2006.

[23] X.-D. Liu and X.-C. Lu. A thermodynamic understanding of clay-swelling inhibition by potassium ions. Angew. Chem. Int. Ed., 45(38):6300-6303, 2006. 
[24] E.J.M. Hensen, T.J. Tambach, A. Bliek, and B. Smit. Adsorption isotherms of water in Li-, Na- and K-montmorillonite by molecular simulation. J. Chem. Phys., 115(7):3322-3329, 2001.

[25] E.J.M. Hensen and B. Smit. Why clays swell. J. Phys. Chem. B, 106(49):12664-12667, 2002.

[26] T.J. Tambach, P.G. Bolhuis, and B. Smit. A molecular mechanism of hysteresis in clay swelling. Angew. Chem. Int. Ed., 43(20):2650-2652, 2004.

[27] T.J. Tambach, P.G. Bolhuis, E.J.M. Hensen, and B. Smit. Hysteresis in clay swelling induced by hydrogen bonding: Accurate prediction of swelling states. Langmuir, 22(3):1223-1234, 2006.

[28] M. L. Schlegel, A. Manceau, D. Chateigner, and L. Charlet. Sorption of metal ions on clay minerals I. Polarized EXAFS evidence for the adsorption of Co on the edges of hectorite particles. J. Coll. Interf . Sci., 215(1):140-158, 1999.

[29] M. L. Schlegel, A. Manceau, L. Charlet, and J. L. Hazemann. Adsorption mechanisms of Zn on hectorite as a function of time, pH, and ionic strength. Am. J. Sci., 301(9):798-830, 2001.

[30] R. Dahn, A.M. Scheidegger, A. Manceau, M. L. Schlegel, B. Baeyens, M.H. Bradbury, and D. Chateigner. Structural evidence for the sorption of Ni(II) atoms on the edges of montmorillonite clay. Geochim. Cosmochim. Acta, 67(1):1-15, 2003.

[31] ANDRA. Évaluation de la faisabilité du stockage géologique en formation argileuse. Dossier 2005 Argile : Synthèse, Châtenay-Malabry, France, 2005.

[32] J.-P. Morel, V. Marry, P. Turq, and N. Morel-Desrosiers. Effect of temperature on the retention of $\mathrm{Cs}^{+}$by Na-montmorillonite: microcalorimetric investigation. J. Materials Chemistry, $17: 2812-2817,2007$.

[33] R. G. Gast. Standard free energy of exchange for alkali metal cations on wyoming bentonite. Soil Sci. Soc. Am. J., 33:37-41, 1969.

[34] R. G. Gast. Alkali metal cation exchange on chambers montmorillonite. Soil Sci. Soc. Am. J., 36:14-19, 1992.

[35] I. Shainberg and W. D. Kemper. Ion exchange equilibria on montmorillonite. Soil Sci., 103:4-9, 1967.

[36] G. Eisenmann. Cation selective glass electrodes and their mode of operation. Biophys. J., 2:259-323, 1962.

[37] D. A. Laird and C. Shang. Relationship between cation exchange selectivity and crystalline swelling in 2:1 expanding phyllosilicates. Clay Clay Miner., 45:681-689, 1997. 
[38] A. Maes and A. Cremers. Highly selective ion exchanger in clay minerals and zeolites, j. a. david and k. f. hayes (ed). In Geochemical processes at mineral surfaces, pages 254-295. American Chemical Society, Washington D.C., 1986.

[39] G. Sposito. The surface chemistry of soils. Oxford University Press, 1984.

[40] B. J. Teppen and D. M. Miller. Hydration energy determines isovalent cation exchange selectivity by clay minerals. Soil Sci. Soc. Am. J., 70(1):31-40, 2006.

[41] J. G. Kirkwood. Statistical mechanics of fluid mixtures. J. Chem. Phys., 3(5):300-313, 1935.

[42] D. Frenkel and B. Smit. Understanding Molecular Simulations, From Algorithms to Applications. Academic Press, 2002.

[43] E. Maegdefrau and U. Hoffman. Die Kristallstruktur des Montmorillonits. Z. Kristallogr. Kristallgeom. Kristallphys. Kristallchem., 98:299-323, 1937.

[44] H.J.C Berendsen, J.R. Grigera, and T.P. Straatsma. The missing term in effective pair potentials. J. Phys. Chem., 91(24):6269-6271, 1987.

[45] J. Åqvist. Ion-water interaction potentials derived from free energy perturbation simulations. J. Phys. Chem., 94:8021-8024, 1990.

[46] D.E. Smith. Molecular computer simulations of the swelling properties and interlayer structure of cesium montmorillonite. Langmuir, 14(20):5959-5967, 1998.

[47] M. Sprik and R. Vuilleumier. Electronic structure and solvation of copper and silver ions: A theoretical picture of a model aqueous reaction. J. Am. Chem. Soc., 126(12):3928-3938, 2004.

[48] M. A. Burgess. Metal Ions in Solution. Ellis Horwood, 1978.

[49] Y. Marcus. The thermodynamics of solvation of ions. part 2. The enthalpy of hydration at 298.15K. J. Chem. Soc. Faraday Trans., 83(2):339-349, 1987.

[50] Y. Marcus. Thermodynamics of solvation of ions. part 5. Gibbs free energy of hydration at 298.15K. J. Chem. Soc. Faraday Trans., 87(18):2995-2999, 1991.

[51] V. Marry and P. Turq. Microscopic simulations of interlayer structure and dynamics in bihydrated heteroionic montmorillonites. J. Phys. Chem. B, 107(8):1832-1839, 2003.

[52] C. Liu, J. M. Zachara, O. Qafoku, and S. C. Smith. Effect of temperature on Cs ${ }^{+}$sorption and desorption in subsurface sediments at the Hanford site, USA. Env. Sci. Tech., 37(12):2640$2645,2003$.

[53] Tertre E., G. Berger, S. Castet, M. Loubet, and E. Giffaut. Experimental sorption of $\mathrm{Ni}^{2+}, \mathrm{Cs}^{+}$ and $\mathrm{Ln}^{3+}$ onto a montmorillonite up to $150^{\circ}$ C. Geochim. Cosmochim. Acta, 69(21):4937-4948, 
2005.

[54] W. Kunz, P. Lo Nostro, and B. W. Ninham. The present state of affairs with Hofmeister effects. Curr. Opinion Coll. Interf. Sci., 9(1-2):1 - 18, 2004.

[55] B. Rotenberg, V. Marry, R. Vuilleumier, N. Malikova, C. Simon, and P. Turq. Water and ions in clays : Unraveling the interlayer/micropore exchange using molecular dynamics. Geochim. et Cosmochim. Acta, 71:5089-5101, 2007. 
FIG. 1: Thermogram obtained upon the successive immersions of three $\mathrm{MNa}(\mathrm{RH}=43 \%)$ samples in water. Peak c: calibration. Peak 1: $13.0 \mathrm{mg}$ yields $\Delta_{i} H^{\mathrm{Na}}=23.9 \mathrm{~mJ} / \mathrm{mg}$. Peak 2: $17.0 \mathrm{mg}$ yields $\Delta_{i} H^{\mathrm{Na}}=23.2 \mathrm{~mJ} / \mathrm{mg}$. Peak $3: 23.4 \mathrm{mg}$ yields $\Delta_{i} H^{\mathrm{Na}}=23.5 \mathrm{~mJ} / \mathrm{mg}$.

FIG. 2: The simulation box contains to Montmorillonite layers, one in the center, the other cut in two halves at the top and bottom of the box, and corresponds to a periodic stack of infinite layers. Counterions (blue) and water molecules are in the interlayer space. $\mathrm{Al}$ atoms are represented in green, $\mathrm{Si}$ atoms in yellow, $\mathrm{O}$ atoms in red and $\mathrm{H}$ atoms in white.

FIG. 3: Average $\langle\partial \mathcal{V} / \partial \lambda\rangle$ as a function of $\lambda$, for the thermodynamic integration from $\mathrm{Na}^{+}$ to $\mathrm{Cs}^{+}$in aqueous solution (solid line) and in clay with $n_{H 2 O}=6$ water molecules per cation (dashed line). Standard deviations are smaller than the symbols. The integrals are the difference in hydration free energy $\Delta_{h} F$ and the enthalpy for the exchange of ions between clay and the gas phase $\Delta_{r} F_{g}^{0}(6)$, respectively. 
Figure 1

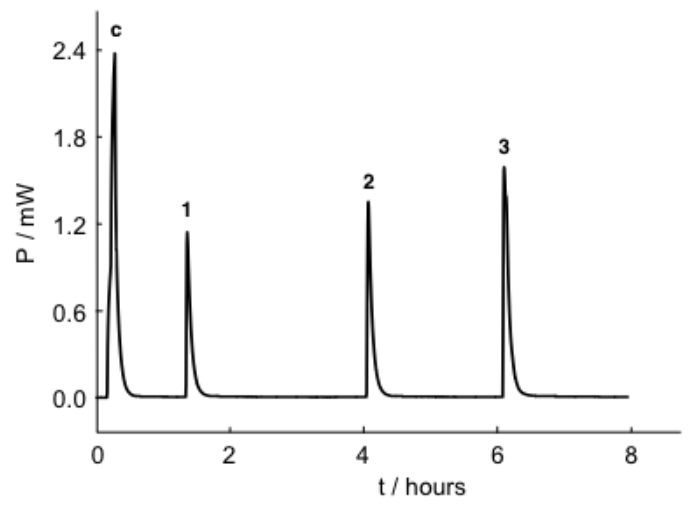


Figure 2

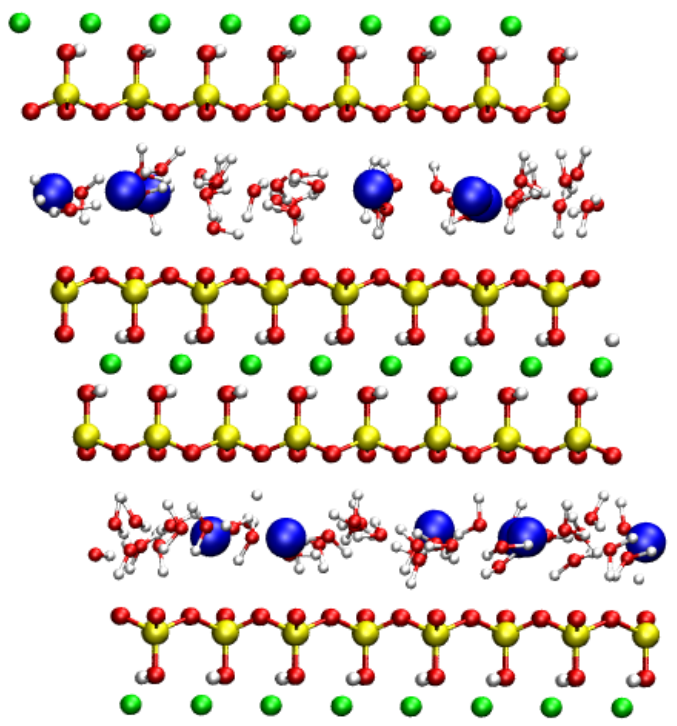


Figure 3

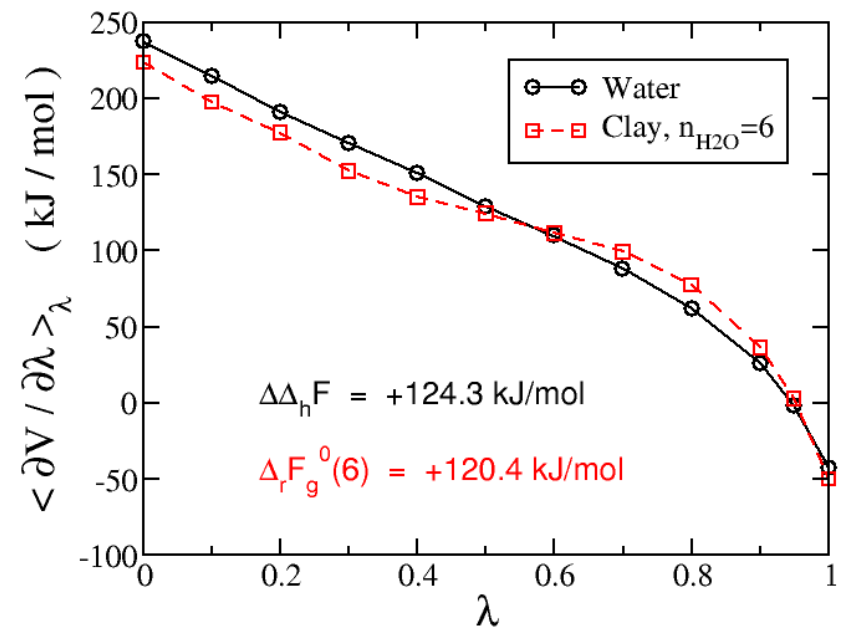

